\title{
REFLECTIVE FUNCTORS IN GENERAL TOPOLOGY AND ELSEWHERE
}

\author{
BY \\ J. F. KENNISON(1)
}

1. Introduction. The motivating problem for this paper is to determine which topological properties, such as compactness, admit operations analogous to the Stone-Čech compactification. To be formal, we shall let $T$ denote the category of topological spaces and continuous functions. We then consider the problem of finding those full subcategories $P$ of $T$ for which the injection functor from $P$ into $T$ has an adjoint $F: T \rightarrow P$ or a coadjoint $G: T \rightarrow P$. A condition equivalent to the existence of such adjoints is mentioned in the paragraph concerning notation in \$1.1. Sometimes the terms "left adjoint" and "right adjoint" are used instead of "adjoint" and "coadjoint," e.g., contrast [8] with [6]. We are indebted to the referee for a number of helpful suggestions, particularly for calling our attention to the following convenient definition. (It is an adaptation of terminology used by Freyd in [3].)

Definition. A category $B$ is reflective in $A$ if $B$ is a full subcategory of $A$ such that the injection functor from $B$ into $A$ has an adjoint $F: A \rightarrow B$. In this case, $F$ is the reflective functor (or reflector) associated with $B$.

Dually, $B$ is coreflective in $A$ if the injection functor admits a coadjoint $G: A \rightarrow B$ known as the coreflective functor (or coreflector) associated with $B$.

Note. We have, for the sake of convenience, modified the usual definition of "reflective subcategory" which would result if the word "full" were deleted from the above definition.

In this paper we shall find conditions on a subcategory $B$ equivalent to the existence of a reflective functor, $F: A \rightarrow B$, which satisfies a certain kind of functorial equation. This result enables us to classify reflective and coreflective subcategories of $T$. All coreflective subcategories are of the same type and Theorem A gives necessary and sufficient conditions for a subcategory of $T$ to be coreflective. Theorems B, C and D describe three types of reflective subcategories. We do not know whether these three types include all of the reflective subcategories of $T$.

Received by the editors February 6, 1964.

(1) This paper is based on the author's Ph.D. thesis submitted to Harvard University. The author wishes to express his thanks to his advisor, A. M. Gleason, for encouragement and direction. 
In the remainder of this section, we shall develop enough terminology to state these main results, for the case of the category $T$. Our most general theorems are in $\S 2$. In $\S 3$ we shall prove Theorems A, B, C and D by showing that they reduce to special cases of the general results of $\$ 2$. By the same method analogous results for uniform spaces can be proven (as indicated in $\$ 3$ ).

Our topological conventions will be based on Kelley's General topology [7]. Thus an object of $T$ is an ordered pair, $(A, L)$, where $L$ is the family of all open subsets of the underlying set $A$.

1.1. Classification of reflective and coreflective functors on $T$.

Notation. Our notation for reflective functors (on any category) will be based on [8]. If $F: A \rightarrow B$ is a reflector then there exists a front adjunction map, as defined in [8] and [6], which will always be denoted herein by $e_{X} \in \operatorname{Hom}(X, F(X))$. The characteristic properties of this map are that $e_{Y} f=F(f) e_{X}$ for all $f \in \operatorname{Hom}(X, Y)$ and, defining $\left(e_{X}\right)^{*}(g)$ as $g e_{X}$, the function $\left(e_{X}\right)^{*}: \operatorname{Hom}(F(X), Q) \rightarrow \operatorname{Hom}(X, Q)$ is one-to-one and onto whenever $Q \in B$. (By ' $Q \in B$,'” we mean that $Q$ is an object of $B$.) The reflectivity of $F$ is equivalent to the existence of such maps $\left\{e_{X}\right\}$. Dually, if $G: A \rightarrow B$ is a coreflector then the end adjunction map will always be denoted herein by $\varepsilon_{X} \in \operatorname{Hom}(G(X), X)$ and we define $\left(\varepsilon_{X}\right)_{*}(g)$ as $\varepsilon_{X} g$.

Definition. Let $F: T \rightarrow P$ be a reflector defined on the category of topological spaces. $F$ is a simple reflector if $e_{X}: X \rightarrow F(X)$ is one-to-one and onto for all $X$.

$F$ is an identifying reflector if $e_{X}$ maps $X$ onto $F(X)$ for all $X$.

$F$ is an embedding reflector if each object of $P$ is a Hausdorff space and if $e_{X}(X)$ is a dense subset of $F(X)$ for all $X$.

Definition. The full subcategory $P$ is simple (resp. identifying, or embedding) iff there exists a simple (resp. identifying, or embedding) reflector $F: T \rightarrow P$.

Definition. A coreflector $G: T \rightarrow P$ is cosimple if $\varepsilon_{X}: G(X) \rightarrow X$ is one-to-one and onto for all $X$.

1.2. Classification of topological properties. By a topological property $P$, we mean a full subcategory of $T$ which is closed under the formation of equivalent (= homeomorphic) objects. (In general such subcategories are called replete.) The following definition extends a definition in Kelley, [7, p. 133].

Definition. A topological property $P$ is hereditary (resp. divisible, productive, or coproductive) if the objects of $P$ are closed under the formation of relative subspaces (resp. quotient spaces, product spaces, or coproduct spaces.)

Note. The terms "quotient space" and "relative subspace" are used in their topological sense which is much more specialized than the category theoretic terms of "quotient object" and "subobject," (cf. the note in \$1.3).

The topological product is equivalent to the category product. The category theoretic coproduct exists in $T$ but seems to have been ignored by Kelley. Conceptually, the coproduct of a family of spaces is simply their disjoint union. To be explicit we shall let $\left\{\left(A_{i}, L_{i}\right)\right\}$ be an indexed family of spaces. We shall assume that $A \cap A=\varnothing$ for $i \neq j$. The conroduct of this family is then (homeo- 
morphic to) the space $(A, L)$, where $A=\bigcup A_{i}$ and $L$ is the topology generated by $\bigcup L_{i}$.

The category theoretic definition of the product and its dual notion of the coproduct (also known as the sum) can be found in [3] or [8]. (Briefly, an object $X$ together with projection morphisms, $p_{i}: X \rightarrow X_{i}$ is a product of the indexed family of objects $\left\{X_{i}\right\}$ iff for each indexed collection $\left\{f_{i}\right\}$ with each $f_{i} \in \operatorname{Hom}\left(Y, X_{i}\right)$, there is a unique $f \in \operatorname{Hom}(Y, X)$ such that $p_{i} f=f_{i}$ for all $i$. We shall often write $X=\prod X_{i}$.)

We shall also classify topological properties by using the following two definitions.

Definition. A topological property $P$ is closed-hereditary if $A \in P$ whenever $A$ is a closed subset (with the relative topology) of some $Q \in P$.

Definition. A topological property $P$ is nontrivial if $P$ contains at least one nonvoid space.

1.3. Statements of main results concerning $T$. Suppose $F: T \rightarrow P$ is a reflector (or coreflector). If we let $P^{\prime}$ be the topological property generated by the full subcategory $P$, then $F: T \rightarrow P^{\prime}$ is still a reflector (or coreflector). Hence we are justified in restricting our attention to those (co)reflective functors $F: T \rightarrow P$ for which $P$ is a topological property.

Theorem A. A topological property $P$ is coreflective iff $P$ is nontrivial, divisible and coproductive. Moreover, every coreflective functor with nontrivial range is cosimple.

THEOREM B. A topological property $P$ is simple iff $P$ is hereditary, productive and contains every indiscrete space.

TheOREM C. A topological property $P$ is identifying iff $P$ is hereditary and productive.

THEOREM D. A topological property $P$ is embedding iff $P$ is closed-hereditary, productive and contains only Hausdorff spaces.

The most famous example of a reflector on $T$ is a probably the Stone-Čech compactification. The Hewitt realcompactification, described in [4], is another embedding reflector. Other reflective properties are formed by the classes of all $T_{0}, T_{1}, T_{2}$, and $T_{3}$ spaces; the class of all regular spaces; the completely regular spaces and even the class of all totally disconnected spaces.

The classes of all locally connected and of all locally arcwise connected spaces form coreflective subcategories (e.g., see [10] and [11]).

Note. Theorem $\mathrm{C}$ generalizes a category theoretic result stated in [3] that $P$ is reflective in $T$ if $P$ is productive and closed under the formation of subobjects. One can show that the classes of all $T_{0}, T_{1}$ and $T_{2}$ spaces are closed under subobject formation whereas the class of regular spaces is not. For example, let $I$ be 
the set of irrational numbers. Let $S$ be the standard topology on the real line, $R$, and let $N$ be the nonstandard topology generated by $S \cup\{I\}$. A basic $N$-open set has the form $(a, b)$ or $(a, b) \cap I$. Now $N$ is not regular since $Q=R-I$ is $N$-closed but $\sqrt{ } 2$ and $Q$ cannot be separated by disjoint $N$-open sets. However, $(R, N)$ is a subobject of $(R, S)$ since the continuous function $1_{R}:(R, N) \rightarrow(R, S)$ is a monic morphism (i.e., left cancellable).

There is a topological characterization of those identifying reflectors $F: T \rightarrow P$ for which $P$ is closed under subobject formation. One can show by closely examining the constructions given in Theorem 2.5 and also in $\$ 3.3$ that $e_{X}: X \rightarrow F(X)$ is always a quotient map in the topological sense iff $P$ is closed under subobject formation iff $P$ is closed under "super-topologies," meaning that if $L$ and $M$ are topologies on $A$ with $L \subseteq M$ and $(A, L) \in P$, then $(A, M) \in P$.

1.4. Preliminary observations. In this section we shall prove a lemma which will enable us, in effect, to make several simplifying assumptions concerning reflective functors.

LeMma 1.1. If $F: A \rightarrow B$ is a reflector and if $Q \in B$, then $e_{Q} \in \operatorname{Hom}(Q, F(Q))$ is an equivalence.

Proof. Let $e=e_{Q}$. Since $e^{*}: \operatorname{Hom}(F(Q), Q) \rightarrow \operatorname{Hom}(Q, Q)$ is onto, there is a $j$ such that $e^{*}(j)=j e=1_{Q}$. On the other hand, $e^{*}(e j)=e j e=e=e^{*}\left(1_{F(Q)}\right)$ and hence $e j=1_{F(Q)}$ as $e^{*}$ is one-to-one (from $\operatorname{Hom}(F(Q), F(Q))$ into $\operatorname{Hom}(Q, F(Q))$.

We observe that there is in general no essential difference among equivalent objects nor among naturally equivalent functors. It is an easy consequence of the above lemma that every reflector, $F: A \rightarrow B$, is naturally equivalent to a functor $F^{\prime}: A \rightarrow B$ for which $F^{\prime}(Q)=Q$ whenever $Q \in B$. In addition one can require that $F^{\prime}$ itself be a reflector and that the front adjunction map from $Q$ into $Q=F^{\prime}(Q)$ be the identity, $1_{Q}$.

Definition. $A$ reflector $F: A \rightarrow B$ is in idempotent form if $F(Q)=Q$ and $e_{Q}=1_{Q}$ for all $Q \in B$.

We have shown that every reflector is naturally equivalent to a reflector in idempotent form. We observe that if $F$ is in idempotent form then $F$ is idempotent in the sense that $F^{2}=F$ on objects and morphisms. (The proof that $F^{2}=F$ for morphisms uses the fact that $F^{2}(f) e_{Q}=e_{P} F(f)$ if $F(f) \in \operatorname{Hom}(Q, P)$.)

2. Pullback stripping functors (P.S. F.'s). In this section we shall introduce the notion of a pullback stripping functor (or P.S.F.). The point of defining a P.S.F. lies in the ability to characterize those reflective functors $F$ for which $H F=H$ whenever $H$ is a P. S. F.

2.1. Basic definitions. We recall that a functor $H$ is called faithful iff $f, g \in \operatorname{Hom}(X, Y)$ and $H(f)=H(g)$ imply $f=g$.

Definition. A category $C$ is said to be fully powered if every indexed family of objects of $C$ has a category theoretic product in $C$. 
Definition. Let $H: C \rightarrow R$ be a faithful functor. Let $A$ be an object of $R$. The category $H^{-1}(A)$ consists of all objects $X$ of $C$ for which $H(X)=A$ and all morphisms $f$ for which $H(f)=1_{A}$.

Clearly, $H^{-1}(A)$ is a subcategory of $C$ but is not necessarily a full subcategory.

Definition. Let $H: C \rightarrow R$ be a faithful functor. Let $X$ and $Y$ be objects of $C$ with $H(X)=A$ and $H(Y)=B$. An $R$-morphism $f \in \operatorname{Hom}(A, B)$ is admissible from $X$ to $Y$ if there exists a morphism $g \in \operatorname{Hom}(X, Y)$ such that $H(g)=f$.

Note. If $H: C \rightarrow R$ is faithful, then $H^{-1}(A)$ is, for all $A \in R$, a partially ordered category (or one in which $\operatorname{Hom}(X, Y)$ always has at most one element). By convention, $X \leqq Y$ means that (in $H^{-1}(A)$ ) $\operatorname{Hom}(X, Y) \neq \varnothing$. Equivalently, $X \leqq Y$ iff $H(X)=H(Y)=A$ and $1_{A}$ is admissible from $X$ to $Y$, for some $A \in R$. Clearly if $X \leqq Y$ and $Y \leqq X$, then $X$ and $Y$ are equivalent as objects of $H^{-1}(A)$. We shall frequently use " $X=Y$ " to mean that $X \leqq Y$ and $Y \leqq X$.

It is standard terminology to define a "small" category as one whose class of objects is a set. By a skeleton $M$ of a category $C$ we mean a full subcategory for which each object of $C$ is equivalent to one and only one object of $M$. We are now ready to make the most important definition of this paper:

Definition: Let $C$ be a fully powered category. A faithful functor $H: C \rightarrow R$ is a pullback stripping functor (or a P. S. F.) if:

1. For each $A \in R$, the category $H^{-1}(A)$ is fully powered and has a small skeleton.

2. For each $R$-morphism $f \in \operatorname{Hom}(A, B)$ and each $Y \in H^{-1}(B)$ there is a largest object $X$ in the partially ordered category $H^{-1}(A)$ such that $f$ is admissible from $X$ to $Y$. ( $X$ is clearly determined up to an $H^{-1}(A)$ equivalence.) $X$ is the $f$-pullback of $Y$ and is denoted by $X=f^{\circ}(Y)$.

3. $g^{0} f^{0}(Y)=(f g)^{0}(Y)$, when defined.

For the remainder of $\S 2$ we shall assume $H: C \rightarrow R$ is a P. S. F.

Remarks. (Let. $H: C \rightarrow R$ be a P.S. F.) We then observe that:

1. $f$ is admissible from $X$ to $Y$ iff $X \leqq f^{0}(Y)$. For if $X \leqq f^{0}(Y)$ and $A=H(X)$ then $1_{A}$ is admissible from $X$ to $f^{0}(Y)$ and so $f=f 1_{A}$ is admissible from $X$ to $Y$.

2. The pullback function $f^{0}: H^{-1}(B) \rightarrow H^{-1}(A)$ is order preserving, in the sense that $f^{0}(Y) \leqq f^{0}(Z)$ if $Y \leqq Z$.

3. If $f=1_{A}$ then $f^{0}: H^{-1}(A) \rightarrow H^{-1}(A)$ is the identity function.

4. In a partially ordered category, the notion of product turns out to be equivalent to the notion of greatest lower bound. Hence a small, partially ordered category is fully powered iff it is a complete lattice. (Note that every "sup" is an "inf" of upper bounds.) It follows that any skeleton of the category $H^{-1}(A)$ is a complete lattice. It is suggestive to denote the $H^{-1}(A)$ category product of an indexed family of objects, $\left\{X_{i}\right\}$, by $\wedge X_{i}$. Of course this " $\wedge$-product" is defined iff there is some $A$ with $H\left(X_{i}\right)=A$ for all $i$.

5. We further note that every class (and not only every set) of objects in $H^{-1}(A)$ has a greatest lower bound (because $H^{-1}(A)$ has a skeleton which is a set, etc.). 
We shall denote the greatest lower bound of a class, $Z \subseteq H^{-1}(A)$, by $\wedge Z$. Setting $Z=H^{-1}(A)$ and $Z=\varnothing$, we see that $H^{-1}(A)$ has minimal and maximal elements.

6. The category $R$ is fully powered. (We shall indicate the proof. Let $\left\{A_{i}\right\}$ be an indexed family of objects of $R$. Let $X_{i}$ be minimal in $H^{-1}\left(A_{i}\right)$ for all $i$. Since $C$ is fully powered, the product $\prod X_{i}$ exists together with projections $p_{j}: \prod X_{i} \rightarrow X_{j}$ for each $j$. It can be shown that $H\left(\prod X_{i}\right)$ together with projections $H\left(p_{j}\right)$ is a product in $R$ for the family $\left\{A_{i}\right\}$.)

We shall write $\prod A_{i}=H\left(\prod X_{i}\right)$ and $q_{j}=H\left(p_{j}\right)$.

2.2. The $\wedge$-product and the $\prod$-product. In this section we shall relate the $\Pi$-product to the $\bigwedge$-product and vice versa. To this end we shall let $\left\{X_{i}\right\}$ be an indexed family of objects of $C$. We shall set $H\left(X_{i}\right)=A_{i}$, for all $i$, and set $B=\prod A_{i}$ with projections $H\left(p_{i}\right)=q_{i}: B \rightarrow A_{i}$. It follows that $q_{i}^{0}\left(X_{i}\right) \in H^{-1}(B)$ for all $i$.

THEOREM 2.1. $\prod X_{i}=\bigwedge q_{i}^{0}\left(X_{\imath}\right)$.

Proof. Since $q_{i}=H\left(p_{i}\right), q_{i}$ is admissible from $\prod X_{i}$ to $X_{i}$. Thus in view of remark 1 of the previous section, $\prod X_{i} \leqq q_{i}^{0}\left(X_{i}\right)$. Since this is true for all $i$, $\prod X_{i} \leqq \wedge q_{i}^{0}\left(X_{i}\right)$.

On the other hand, for each $j, \wedge q_{i}^{0}\left(X_{i}\right) \leqq q_{j}^{0}\left(X_{j}\right)$ and hence (by remark 1 again) $g_{j}$ is admissible from $\wedge q_{i}^{0}\left(X_{i}\right)$ to $X_{j}$. Thus, $q_{j}=H\left(s_{j}\right)$ for some $s_{j}: \wedge q_{i}^{0}\left(X_{i}\right) \rightarrow X_{j}$. Moreover, since $\prod X_{i}$ is a product, there is a unique $s: \wedge q_{i}^{0}\left(X_{i}\right) \rightarrow \prod X_{i}$ for which $p_{j} s=s_{j}$. Setting $m=H(s)$, we obtain $\wedge q_{i}^{0}\left(X_{i}\right) \leqq m^{0} \prod X_{i}$, as $m$ is admissible. We claim that $m=1_{B}$, which in view of remark 3 of the previous section, completes the proof. Observe that $q_{j} m=q_{j}$ as $H\left(p_{j} s\right)=H\left(s_{j}\right)$. Since the $q_{j}$ 's are projections, the equations $q_{j} 1_{B}=q_{j}$ uniquely characterize $1_{B}$ so $m=1_{B}$.

We shall next assume that $\bigwedge X_{i}$ is well defined, and hence that there is some $A$ with $\left\{X_{i}\right\} \subseteq H^{-1}(A)$ (i.e., $H\left(X_{i}\right)=A_{i}=A$ for all $i$ ). Since $\bigwedge X_{i} \leqq X_{j}$ there is a morphism, $d_{j}: \wedge X_{i} \rightarrow X_{j}$, which belongs to the category $H^{-1}(A)$ (or such that $\left.H\left(d_{j}\right)=1_{A}\right)$. In fact, the $d_{j}$ 's are the projections associated with the $\wedge$-product. Since $\prod X_{i}$ is a product, there is a morphism $d: \wedge X_{i} \rightarrow \prod X_{i}$ such that $p_{i} d=d_{i}$ for all $i$. We shall set $d=H(\bar{d})$. Note that $d: A \rightarrow B$. The maps $d$ and $d$ will be called diagonal maps.

TheOREM 2.2. $\bigwedge X_{i}=d^{0} \prod X_{i}$ (if $d$ is the diagonal map defined above).

Proof. $d$ is admissible from $\bigwedge X_{i}$ to $\prod X_{i}$ as $d=H(\bar{d})$ and so $\bigwedge X_{i} \leqq d^{0} \prod X_{i}$. On the other hand, Theorem 2.1 implies $\prod X_{i} \leqq q_{j}^{0}\left(X_{j}\right)$ and hence, as $d^{0}$ is order preserving, $d^{0} \prod X_{i} \leqq d^{0} q_{j}^{0}\left(X_{j}\right)$. But in view of the definition of a P.S.F., $d^{0} q_{j}^{0}\left(X_{j}\right)=\left(q_{j} d\right)^{0} X_{j}=X_{j}$ since $q_{j} d=1_{A} \quad$ (i.e., $\left.q_{j} d=H\left(p_{j} d\right)=H\left(d_{j}\right)=1_{A}\right)$. Thus $d^{0} \prod X_{i} \leqq X_{j}$ for all $j$ and so $d^{0} \prod X_{i} \leqq \bigwedge X_{i}$.

\subsection{H-reflectors.}

Definition. Let $H: C \rightarrow R$ be a P. S. F. A reflector $F: C \rightarrow P$ is an $H$-reflector if $F$ is in idempotent form and $H F=H$ on objects and morphisms. 
If such an $H$-reflector, $F$, exists then $P$ is an $H$-reflective category and is the fixpoint class of $F$.

Remark. Let $F: C \rightarrow P$ be an $H$-reflector. Let $X \in H^{-1}(A)$. Then $H F(X)=H(X)=A$ so $F(X) \in H^{-1}(A)$. Obviously $e_{X}^{*}\left(F\left(e_{X}\right)\right)=F\left(e_{X}\right) e_{X}=e_{F(X)} e_{X}$ $=e_{X}=e_{X}^{*}\left(1_{F(X)}\right)$ and so $F\left(e_{X}\right)=1_{F(X)}$. Moreover, $H\left(e_{X}\right)=H F\left(e_{X}\right)=1_{A}$. Hence $e_{X}$ is a morphism of $H^{-1}(A)$ so $X \leqq F(X)$. We have shown that $F$ is "increasing", on $H^{-1}(A)$.

It is also clear that $F$ is order preserving on $H^{-1}(A)$. For if $X \leqq Y$, there is an $f \in \operatorname{Hom}(X, Y)$ for which $H(f)=1_{A}$. Therefore $H F(f)=H(f)=1_{A}$ and $F(f)$ is a morphism of $H^{-1}(A)$ which indicates that $F(X) \leqq F(Y)$.

THEOREM 2.3. A functor $F: C \rightarrow C$ is an H-reflector iff:

(1) $F$ is idempotent on objects (i.e., $F(F(X))=F(X)$ for all $X \in C)$.

(2) $H F=H$ on objects and morphisms.

(3) $F$ is increasing in the sense that $X \leqq F(X)$ for all $X \in C$.

Proof. In view of the above remark every $H$-reflector satisfies the three conditions. Conversely, assume that $F$ satisfies (1), (2) and (3). Let $X \in H^{-1}(A)$ be arbitrary. $F(X) \in H^{-1}(A)$ and $X \leqq F(X)$ and so there is a map $e_{X} \in \operatorname{Hom}(X, F(X))$ such that $H\left(e_{X}\right)=1_{A}$. Let $P$ be the full subcategory generated by the range of $F$. If $Q \in P$ then $F(Q)=Q$ and $e_{Q}=1_{Q}$ since $H\left(e_{Q}\right)=H\left(1_{Q}\right)$ and $H$ is faithful.

We claim that if $f \in \operatorname{Hom}(X, Y)$ then $F(f) e_{X}=e_{Y} f$. But $H\left(F(f) e_{X}\right)=H F(f) H\left(e_{X}\right)$ $=H F(f)=H(f)=H\left(e_{Y}\right) H(f)=H\left(e_{Y} f\right)$. The claimed equation follows since $H$ is faithful. Moreover, if $Y \in P$ then $e_{Y}=1_{Y}$ and so $f=F(f) e_{X}=e_{X}^{*} F(f)$ proving that $e_{X}^{*}$ maps $\operatorname{Hom}(F(X), Y)$ onto $\operatorname{Hom}(X, Y)$. Finally if $f=e_{X}^{*}(g)=g e_{X}$ then $H F(f)=H(f)=H(g)$ and hence $g=F(f)$ and so $e_{X}^{*}$ is one-to-one. It follows that $F$ is an $H$-reflector.

2.4. $H$-reflective categories. It is easy to show that if $P$ is $H$-reflective then the replete full subcategory generated by $P$ is also $H$-reflective. We shall restrict our attention to those $H$-reflective categories which are replete in $C$.

Definition. Let $P$ be a full subcategory replete in $C$. Then $P$ is a pullback category if $f^{0}(Q) \in P$ whenever $Q \in P$ and $f^{0}(Q)$ is meaningful.

Definition. A category $P$ is $\bigwedge$-productive if $Q_{i} \in P \cap H^{-1}(A)$ for all $i$ implies $\wedge Q_{i} \in P$.

$P$ is $\prod$-productive if $Q_{i} \in P$ for all $i$ implies $\prod Q_{i} \in P$.

In view of Theorems 2.1 and 2.2 , a pullback category $P$ is $\wedge$-productive iff it is $\prod$-productive. Such a category is a productive pullback category.

THEOREM 2.4. If $P$ is an $H$-reflective category, replete in $C$, then $P$ is a productive pullback category.

Proof. Let $F: C \rightarrow P$ be the associated $H$-reflector. Suppose that $Q \in P$ and $f^{0}(Q)$ is meaningful. Since $f$ is admissible from $f^{0}(Q)$ to $Q, f=H(g)$ for some 
$g: f^{0}(Q) \rightarrow Q$. Hence $F(g): F\left(f^{0}(Q)\right) \rightarrow Q$ and since $H F(g)=f, f$ is admissible from $F\left(f^{0}(Q)\right)$ to $Q$. Hence $F\left(f^{0}(Q)\right) \leqq f^{0}(Q)$, and so $F\left(f^{0}(Q)\right)=f^{0}(Q)$ as $F$ is increasing. Thus $f^{0}(Q) \in P$ and $P$ is a pullback category.

It suffices to show that if $Q_{i} \in P \cap H^{-1}(A)$ for all $i$ then $\wedge Q_{i} \in P$. Since $F$ is order preserving $F\left(\wedge Q_{i}\right) \leqq F\left(Q_{i}\right)=Q_{i}$ for all $i$ and so $F\left(\wedge Q_{i}\right) \leqq \wedge Q_{i}$. Hence $F\left(\bigwedge Q_{i}\right)=\bigwedge Q_{i}$, and $\wedge Q_{i} \in P$.

THEOREM 2.5. If $P$ is a productive pullback category then $P$ is an H-reflective category.

Proof. Let $X \in C$ be arbitrary with $A=H(X)$. In view of remark 5 of $\$ 2.1$ we can define $F(X)=\bigwedge\left\{Q \in H^{-1}(A) \mid X \leqq Q\right.$ and $\left.Q \in P\right\}$. It is easy to verify that $X \in P$ iff $X=F(X)$ and that $F$ is increasing and idempotent (i.e., $X \leqq F(X)=F(F(X))$ ).

Next, consider an arbitrary $g \in \operatorname{Hom}(X, Y)$. Since $f=H(g)$ is admissible, $X \leqq f^{0}(Y)$, hence $X \leqq f^{0}(F(Y))$ as $f^{0}$ is order preserving. But $f^{0}(F(Y)) \in P$ and so by the construction of $F$ it follows that $F(X) \leqq f^{0}(F(Y))$. Therefore $f$ is admissible from $F(X)$ to $F(Y)$ and hence $f=H(h)$ for a unique $h \in \operatorname{Hom}(F(X), F(Y)$ ). Clearly $F$ can be regarded as a functor from $C$ onto $P$ if we define $F(g)=h$. By Theorem 2.3, $F$ is an $H$-reflector and so $P$ is an $H$-reflective category.

2.5. Maximal objects and maximal subobjects. In this section we shall sharpen the criterion for reflectivity given in Theorems 2.4 and 2.5.

Definition. $X \in C$ is a maximal object if $X$ is maximal in the partially ordered category $H^{-1}(A)$, where $A=H(X)$.

Definition. $Y$ is a maximal subobject of $X$ iff $Y=f^{0}(X)$ for some monic (i.e., left-cancellable) morphism $f$ of $R$.

Definition. A full subcategory $P$ of $C$ is $H$-maximal if $X \in P$ for all maximal objects $X$.

$P$ is $H$-hereditary if $P$ is closed under the formation of maximal subobjects.

LemMa 2.6. If $X$ is a maximal object then $f^{\circ}(X)$ is also maximal.

Proof. $X=\wedge \varnothing$ (the $\wedge$-product over the null set) since $X$ is maximal. By Theorem 2.2, $X=d^{0} \prod \varnothing$ hence $f^{\circ}(X)=(d f)^{0} \Pi \varnothing$. Since $d f$ is also a diagonal map, $(d f)^{\circ} \prod \varnothing=\wedge \varnothing$ and this is maximal.

Lemma 2.7. Let $P$ be a $\prod$-productive, $H$-maximal subcategory of $C$. Let $q_{j}: \prod A_{i} \rightarrow A_{j}$ be a projection of $R$. If $Q \in P$ and $H(Q)=A_{j}$ then $q_{j}^{0}(Q) \in P$.

Proof. Let $X_{i}$ be maximal in $H^{-1}\left(A_{i}\right)$, for all $i \neq j$. Let $X_{j}=Q$. Then $X_{i} \in P$ for all $i$ so $\prod X_{i} \in P$. In view of Theorem 2.1, $\prod X_{i}=\wedge q_{i}^{0}\left(X_{i}\right)=q_{j}^{0}(Q)$ since $q_{i}^{0}\left(X_{i}\right)$ is maximal for all $i \neq j$.

Clearly, every $H$-reflective category is $\prod$-productive, $H$-hereditary and $H$ maximal (since every maximal object is equivalent to a $\Lambda$-product over the null set). For certain categories $R$, the converse is true as we shall demonstrate. 
Definition. A category $R$ is decomposable if each morphism $h$ of $R$ is a composition, either $h=q_{j} f$ or $h=f q_{j}$, of a projection $q_{j}: \prod X_{i} \rightarrow X_{j}$ and a monic morphism $f$.

TheOREM 2.8. Let $H: C \rightarrow R$ be a P. S. F. for which $R$ is decomposable. $A$ replete full subcategory $P$ of $C$ is $H$-reflective iff $P$ is $\prod$-productive, $H$-hereditary and H-maximal.

Proof. It suffices to show that if $P$ is $\prod$-productive, $H$-hereditary and $H$ maximal then $P$ is a pullback category. Assume $Q \in P$ and $h^{0}(Q)$ is well defined. Since $R$ is decomposable we may as well assume that $h=q_{j} f$, where $f$ is monic and $q_{j}$ is a projection (a similar proof works for $h=f q_{j}$ ). Then $h^{0}(Q)=\left(q_{j} f\right)^{0} Q$ $=f^{0}\left(q_{j}^{0}(Q)\right)$. By Lemma 2.7, $q_{j}^{0}(Q) \in P$. Since $P$ is $H$-hereditary $f^{0}\left(q_{j}^{0}(Q)\right) \in P$.

3. Specific examples of P.S.F.'s and applications. In this section we shall exhibit four P.S. F.' swhich will be denoted by $s^{\prime}, s, H$ and $J$. By applying the theorems of $\S 2$, we shall obtain proofs of our main results (Theorems A, B, C and D). Some concluding observations concerning uniform spaces and other categories will be included in this section.

3.1. The functor $s: T \rightarrow S$. We let $S$ denote the category of sets and functions. If $(A, L)$ is a topological space we define $s(A, L)=A$. If $f:(A, L) \rightarrow(B, M)$ is continuous we define $s(f)=f$. It is entirely straightforward to show that $s$ is a P. S. F. The main steps in the verification are:

(1) A function is admissible iff it is continuous.

(2) $(A, L) \leqq(A, M)$ iff $1_{A}$ maps $(A, L)$ continuously onto $(A, M)$ iff $L \geqq M$. Hence the partially ordered category $s^{-1}(A)$ is equivalent to the complete lattice of all topologies on the set $A$ (with the opposite of the usual ordering). Thus $s^{-1}(A)$ is well powered.

(3) If $f: A \rightarrow B$ is a function and if $M$ is a topology on $B$ then $f^{\circ}(B, M)=(A, L)$, where $L=\left\{V \subseteq A \mid V=f^{-1}(U)\right.$ for some $\left.U \in M\right\}$.

It is easy to show that $f: A \rightarrow B$ is monic in $S$ iff $f$ is one-to-one. If $f$ is monic then $f^{\circ}(B, M)$ is easily shown to be homeomorphic to the relative topology induced by $M$ on $f(A)$. Hence the $s$-hereditary replete full subcategories are the hereditary ones. It is also clear that the $s$-maximal objects are the indiscrete spaces.

Since $S$ is a decomposable category, Theorem 2.8 is applicable. Theorem B thus reduces to the statement that $F$ is a simple reflector iff $F$ is equivalent to an $s$-reflector. But this is obviously the case for if $e_{X}: X \rightarrow F(X)$ is one-to-one and onto then $s\left(e_{X}\right)$ is an equivalence in $S$ between $s(X)$ and $s(F(X))$. Hence we may as well assume that $s(X)=s(F(X))$ and that $s\left(e_{X}\right)=1_{s(X)}$.

3.2. The functor $s^{\prime}: T^{\prime} \rightarrow S^{\prime}$. We shall let $T^{\prime}$ denote the category which is the opposite (or dual) of $T$ and let $S^{\prime}$ denote the opposite of $S$. We define $s^{\prime}: T^{\prime} \rightarrow S^{\prime}$ by setting $s^{\prime}=s$ on objects and maps. The main steps in showing that $s^{\prime}$ is a P.S.F. are: 
(1) An $S^{\prime}$-morphism $f$ is admissible from $X$ to $Y$ iff $f$ is a continuous function from $Y$ to $X$.

(2) $(A, L) \leqq(A, M)$ iff $1_{A}$ maps $(A, M)$ continuously onto $(A, L)$ iff $L \leqq M$. Hence the category $\left(s^{\prime}\right)^{-1}(A)$ is the opposite of $s^{-1}(A)$ and is therefore equivalent to the complete lattice on all topologies on the set $A$, with the usual ordering $\subseteq$.

(3) Let $f$ be an $S^{\prime}$ morphism from $A$ to $B$, then $f: B \rightarrow A$ is a function. Let $(B, M)$ be given. Using $f_{0}$ for the $s^{\prime}$-pullback, we have $f_{0}(B, M)=(A, L)$, where $L=\left\{V \subseteq A \mid f^{-1}(V) \in M\right\}$.

We observe that $f$ is monic in $S^{\prime}$ iff $f$ is onto. If $f$ maps $B$ onto $A$ and if $f_{0}(B, M)=(A, L)$ then $L$ is the quotient topology on $A$ induced by $f$ and $M$. Hence the $s^{\prime}$-hereditary properties are the divisible ones. It is clear also that the $s^{\prime}$-maximal objects are the discrete spaces.

It is easy to show that $S^{\prime}$ is decomposable and that $G: T \rightarrow P$ is cosimple iff $G$ is equivalent to an $s^{\prime}$-reflector. Hence, in view of Theorem 2.8, Theorem A reduces to the following two lemmas.

Lemma 3.1. A nontrivial, divisible and coproductive topological property $P$ contains all discrete spaces.

Proof. Since $P$ is nontrivial and divisible, $P$ contains one-point spaces. Every discrete space, however, is equivalent to a coproduct of one-point spaces.

LEMMA 3.2. Every coreflective functor, $G: T \rightarrow P$, is cosimple, if $P$ is nontrivial

Proof. It is easy to show that $P$ must be nontrivial. Let $Q \in P$ be an object for which $s(Q) \neq \varnothing$. Let $\varepsilon=\varepsilon_{X}: G(X) \rightarrow X$ be an arbitrary end adjunction map. Assume that $\varepsilon(a)=\varepsilon(b)$ with $a \neq b$. Define $f: Q \rightarrow G(X)$ by $f(q)=a$ for all $q \in Q$ and define $g: Q \rightarrow G(X)$ by $g(q)=b$ for all $q \in Q$. Then $(\varepsilon)_{*} g=\varepsilon g=\varepsilon f=\varepsilon_{*} f$, a contradiction, showing $\varepsilon$ is one-to-one.

Next assume that $x \notin \varepsilon(G(X))$. Then if $h: Q \rightarrow X$ is defined by $h(q)=x$ for all $q$ it is clear that $h \neq \varepsilon_{*}(m)$ for any $m \in \operatorname{Hom}(Q, G(X))$. This contradiction shows $\varepsilon$ is onto.

3.3. The functor $H: C \rightarrow S$. The P.S. F. constructed in this section shall be used to prove Theorem $\mathrm{C}$. We need the following definitions.

Definition. The identification category, $C$, has for objects the class of all ordered triples $(A, e, X)$, where $A$ is a set, $X$ is a topological space and $e$ is a function mapping $A$ onto $X$ (or more precisely, perhaps, $e$ maps $A$ onto $s(X)$ ).

A morphism $g:(A, e, X) \rightarrow(B, f, Y)$ is a function from $A$ into $B$ for which there exists a continuous map $\bar{g}: X \rightarrow Y$ such that $\bar{g} e=f g \cdot(\bar{g}$ is determined by $g$ since $e$ is onto and hence right-cancellable.)

Definition. $H: C \rightarrow S$ is the functor for which $H(A, e, X)=A$ and $H(g)=g$. The main steps in showing that $H$ is a P.S.F. are:

(1) $(A, e, X) \leqq(A, f, Y)$ iff $1_{A}$ is admissible iff there exists a continuous function $\bar{g}: X \rightarrow Y$ such that $\bar{g} e=f$. 
(2) The pullback map is defined as follows: Let $g: A \rightarrow B$ be a function and let $(B, f, Y) \in C$. Define $e=f g$. Let $X$ be the space having the relative topology on $e(A) \subseteq Y$. Clearly $(A, e, X) \in C$ and $g$ is admissible from $(A, e, X)$ to $(B, f, Y)$. Moreover, if $g$ is also admissible from $\left(A, e^{\prime}, X^{\prime}\right)$ to $(B, f, Y)$ then there exists a $\bar{g}: X^{\prime} \rightarrow Y$ such that $\bar{g} e^{\prime}=f g=e$. Since $e^{\prime}$ is onto, $\bar{g}\left(X^{\prime}\right) \subseteq X$. Thus $\left(A, e^{\prime}, X^{\prime}\right)$ $\leqq(A, e, X)$ and so we are justified in setting $(A, e, X)=g^{\circ}(B, f, Y)$.

(3) $H^{-1}(A)$ has a small skeleton for all sets $A$. For if $(A, e, X) \in H^{-1}(A)$ then the cardinal of the set $s(X)$ is bounded as $e$ is onto. Hence there is a limit to the number of topologically distinct spaces $X$ for which $(A, e, X)$ can be in $H^{-1}(A)$. For each such space $X$ there is a limited number of maps $e: A \rightarrow X$ and hence the cardinal of any skeleton of $H_{-}{ }^{1}(A)$ is bounded.

(4) $C$ is fully powered with $\prod\left(A_{i}, e_{i}, X_{i}\right)=(A, e, X)$, where $A=\prod A_{i}$ and $X=\prod X_{i}$ (with the product topology). $e$ is the map for which $e_{i} q_{i}=p_{i} e$, where $p_{i}: X \rightarrow X_{i}$ and $q_{i}: A \rightarrow A_{i}$ are the projections in the categories $T$ and $S$.

(5) The $\wedge$-product on $H^{-1}(A)$ is given by the formula which appears in the statement of Theorem 2.2 .

We note that $(A, e, X)$ is $H$-maximal iff $X$ is a one-point space.

Proof of Theorem C. We shall sketch the proof. Let $F: T \rightarrow P$ be an identifying reflector. Let $\bar{P}$ be the full subcategory of $C$ generated by all objects $(A, e, X)$ for which $X \in P$. We define a reflector $F: C \rightarrow \bar{P}$ by setting $F(A, e, X)=\left(A, e_{X} e, F(X)\right)$, where $e_{X}: X \rightarrow F(X)$ is the front adjunction map which is onto. It can easily be shown that if $g$ is admissible from $(A, e, X)$ to $(B, f, Y)$ then $g$ is still admissible from $F(A, e, X)$ to $F(B, f, Y)$. Hence $F$ can be regarded as a functor from $C$ to $\bar{P}$ for which $H F=H$. In view of Theorem $2.3, F$ is an $H$-reflector. Thus $\bar{P}$ is productive and $H$-hereditary implying that $P$ is productive and hereditary (assuming $P$ is replete).

Conversely, let $P$ be a hereditary and productive topological property. Then, using the above definition, $\bar{P}$ is a productive pullback subcategory of $C$. (Productivity of $\bar{P}$ follows from (4) above and pullbacks from (2). Hence, applying Theorem 2.5 there is a $H$-reflector $\bar{F}: C \rightarrow \bar{P}$.

We can now define a reflector $F: T \rightarrow P$. Let $X=(A, L) \in T$. Then $\left(A, 1_{A}, X\right) \in C$. Let $\bar{F}\left(A, 1_{A}, X\right)=\left(A, e_{X}, Y\right)$ and set $F(X)=Y$. The maps $e_{X}: X \rightarrow F(X)$ are continuous and onto and they set up a front adjunction showing that $F$ is an identifying reflector. Hence $P$ is an identifying category.

3.4. The functor $J: E \rightarrow S$.

DEFINITION. The embedding category, $E$, has for objects the class of all ordered triples $(A, e, X)$ for which $A$ is a set, $X$ is a Hausdorff space, $e$ is a function from $A$ into $X$ and $e(A)$ is a dense subset of $X$. A morphism $g:(A, e, X) \rightarrow(B, f, Y)$ is a function from $A$ into $B$ for which there is a continuous map $\bar{g}: X \rightarrow Y$ such that $\bar{g} e=f g$. We note that $\bar{g}$ is uniquely determined on $e(A)$ and since $Y$ is Hausdorff, $\bar{g}$ is uniquely determined on all of $X$.

Definition. $J: E \rightarrow S$ is the functor for which $J(A, e, X)=A$ and $J(g)=g$. 
The main steps in showing that $J$ is a P.S. F. (cf. §3.3) are:

(1) $(A, e, X) \leqq(A, f, Y)$ iff there is a continuous $\bar{g}: X \rightarrow Y$ such that $\bar{g} e=f$.

(2) Let $g: A \rightarrow B$ be a function and let $(B, f, Y) \in C$. Define $e=f g$. Let $X$ be the space having the relative topology on the closure of $e(A)$. Then $f^{0}(B, f, Y)=(A, e, X)$.

(3) $J^{-1}(A)$ always has a small skeleton. For if $(A, e, X) \in J^{-1}(A)$ then the cardinal of $X$ is bounded since $X$ is Hausdorff and has a dense subset, $e(A)$, of cardinal no greater than the cardinal of $A$. The argument used for $H^{-1}(A)$ in the previous section now applies.

(4) $E$ is fully powered with $\prod\left(A_{i}, e_{i}, X_{i}\right)=(A, e, X)$, where $X=\prod X_{i}$, etc. (i.e., with the same construction as in $\S 3.3$ ).

(5) The $\wedge$-product on $J^{-1}(A)$ is given by the formula which appears in Theorem 2.2.

Proof of Theorem D. If $P$ is an embedding property, we then let $\bar{P}$ denote the full subcategory of $E$ containing all $(A, e, X)$ for which $X \in P$. It is easy to show that $\bar{P}$ is a $J$-reflective category and hence is productive and $J$-hereditary (cf. §3.3). In view of (4) and (2), $P$ is productive and closed-hereditary.

Conversely, if $P$ is productive and closed-hereditary then $\bar{P}$ is a productive pullback subcategory of $E$. Hence there is a $J$-reflector; $F: E \rightarrow \bar{P}$. We now let $T_{2}$ be the full subcategory of $T$ containing all Hausdorff spaces. Then $X=(A, L) \in T_{2}$ iff $\left(A, 1_{A}, X\right) \in E$. We can clearly define $F: T_{2} \rightarrow P$ so that $\bar{F}\left(A, 1_{4}, X\right)=(A, e, F(X))$. Moreover, in view of Theorem $\mathrm{C}$ there is an identitifying reflector $G: T \rightarrow T_{2}$ since $T_{2}$ is hereditary and productive. $F G: T \rightarrow P$ is then an embedding functor.

3.5. Uniform spaces. If $U$ denotes the category of uniform spaces and uniform maps then the forgetful functor from $U \rightarrow S$ is a P. S. F. Hence the analogue of Theorem B holds for uniform properties. By using constructions of the type found in $\S \S 3.2,3.3$ and 3.4, one can show that the analogues of Theorems A, C and $\mathrm{D}$ also hold for $U$. The subcategory of all totally bounded (or precompact) uniform spaces is a simple reflective property. The complete and the compact uniform spaces both form embedding properties. The composition of the totally bounded reflector followed by the completion functor gives the Samuel uniform compactification, which is examined in [9].

3.6. Analogous results. Some analogous results concerning the existence of reflectors may be found in the exercises of [3] starting on p. 74. Some of these results are category theoretic versions of theorems about universal algebra proved by Garrett Birkhoff (see [1]).

Theorem D recalls another early result of Mr. Birkhoff's. In [2], he shows, in effect, that for certain general types of reflectors $F: A \rightarrow B$ the morphism $e_{X}$ can be regarded as a function continuously mapping " $X$ " onto a dense subset of " $F(X)$ " (i.e., there is a forgetful functor $H: A \rightarrow S$ and $F$ induces a closure oper- 
at or on $H(X)$ for each object $X \in A)$. Conversely, certain closure operators give rise to reflectors. In the category of completely regular Hausdorff spaces the topological closure operator gives rise to the Stone-Čech compactification.

\section{REFERENCES}

1. G. Birkhoff, Lattice theory, Amer. Math. Soc. Colloq. Publ. Vol. 25, Providence, R. I., 1940; rev. ed., 1948; reprinted, 1964.

2. - The meaning of completeness, Ann. of Math. (2) 38 (1937), 57-60.

3. P. Freyd, Abelian categories, an introduction to the theory of functcrs, Harper and Row New York, 1964.

4. L. Gillman and M. Jerison, Rings of continuous functions, Van Nostrand, Princeton, N. J., 1960.

5. R. Godement, Théorie des faisceaux, Hermann, Paris, 1958.

6. D. Kan, Adjoint functors, Trans. Amer. Math. Soc. 87 (1958), 294-329.

7. J. Kelley, General topology, Van Nostrand, Princeton, N. J., 1955.

8. S. MacLane, Categorical algebra, Amer. Math. Soc. Colloquium Lectures, Proceedings of the Summer Seminar, Univ. of Colorado, Boulder, Col., 1963.

9. P. Samuel, Ultrafilters and compactification of uniform spaces, Trans. Amer. Math. Soc. 64 (1948), 100-132.

10. G. Young, The introduction of local connectivity by change of topology, Amer. J. Math. 68 (1946), 476-494.

11. A. M. Gleason, Universal locally connected refinements, Illinois J. Math.7(1963), 521-531.

\section{Clark University,}

WORCESTER, MASSACHUSETTS 